\section{Collaboration - no other way to go}

$\mathrm{R}$ espiratory disease is ubiquitous and does not take on a single form or affect a select population. The burden of respiratory disease is experienced all over the world, by young and old. If we expect to have an impact on respiratory health, we cannot go it alone - and we don't.

The Canadian Thoracic Society (CTS) has a wide range of partnerships that enables it to maximize its impact in strategic areas (research, education, guidelines and knowledge translation). In addition to collaborating with the Canadian Lung Association, the Canadian Respiratory Health Professionals, the Canadian COPD Alliance, and provincial lung associations and thoracic societies, the CTS works with a wide range of stakeholders and organizations, in Canada and abroad.

Only a few years ago, we welcomed a national group of pediatric respirologists who formed a pediatric assembly (PA) within the CTS. Since then, we've been working on integrating their perspective by appointing pediatric respirologists to many of our committees, creating a pediatric track as part of the Canadian Respiratory Conference (CRC) and including the PA chair (currently Dr Hans Pasterkamp) in our meetings with American Thoracic Society (ATS) leaders.

We collaborate a great deal with our counterparts in the United States and Europe. We meet regularly with American College of Chest Physicians (ACCP), the ATS and European Respiratory Society leaders to exchange information on a wide range of respiratory issues and to plan our collaboration on specific initiatives. Current joint projects include the following: North American collaboration on World Spirometry Day; the development of joint guidelines on acute exacerbations of chronic obstructive pulmonary disease with the ACCP; the delivery of our annual CTS Scientific Program in conjunction with the ACCP CHEST Conference; our collaboration on scientific presentations at conferences such at the CRC and the World Asthma Congress; the delivery of the CTS Research Poster Competition as part of the ATS's annual international conference; and the CTS/ European Respiratory Society Peter Macklem Fellowship exchange program. We will also soon celebrate the inauguration of our own Darcy Marciniuk, a former CTS president, as ACCP president - that is quite a testament to our collaboration on the North American stage!

We are also actively involved in global initiatives such as the Guidelines International Network and the Global Alliance Against Respiratory Disease. We have been well represented there by senior CTS leaders including Louis-Philippe Boulet, Chair of the Canadian Respiratory Guidelines Network.

Our partnerships extend all the way to our Journal. Thanks to the leadership of Peter Paré, Editor-in-Chief of the Canadian Respiratory Journal, we are developing memoranda of understanding with the Canadian Critical Care Society and the Canadian Sleep Society. Through these agreements, we aim to address a wider range of clinical and research issues in the Journal and, thus, enhance the content and readership of the Canadian Respiratory Journal.

Last, but certainly not least, are the partnerships we are nurturing through the National Respiratory Research Strategy (NRRS). Our Faculties of Medicine and division directors from across the country, the Canadian Institutes of Health Research - Institute of Circulatory and Respiratory Health, and AllerGen have been some of the key

\section{La collaboration - la seule issue possible}

a maladie respiratoire est omniprésente, prend plusieurs formes et
touche diverses populations. Son fardeau est ressenti partout dans
le monde, par des jeunes et des moins jeunes. Pour améliorer la santé
respiratoire, il ne faut pas faire cavalier seul... et nous nous en gardons
bien.

La Société canadienne de thoracologie (SCT) entretient un vaste éventail de partenariats qui lui permettent d'optimiser ses répercussions dans des domaines stratégiques (recherche, formation, lignes directrices et transfert du savoir). En plus de collaborer avec l'Association pulmonaire du Canada, les Professionnels canadiens en santé respiratoire, l'Alliance canadienne sur la MPOC ainsi que des associations pulmonaires et sociétés de thoracologie provinciales, la SCT collabore avec une grande diversité d'intervenants et d'organismes, au Canada et à l'étranger.

Il y a quelques années, nous avons accueilli un groupe national de pneumologues pédiatriques qui ont formé une assemblée pédiatrique (AP) au sein de la SCT. Depuis, nous cherchons à intégrer leurs points de vue : nous avons nommé des pneumologues pédiatriques au sein de bon nombre de nos comités, avons créé un volet pédiatrique au Congrès canadien sur la santé respiratoire (CCSR) et invitons le président de l'AP (actuellement le docteur Hans Pasterkamp) à nos réunions avec des dirigeants de l'American Thoracic Society (ATS).

Nous multiplions les collaborations avec nos homologues des ÉtatsUnis et de l'Europe. Nous rencontrons régulièrement des dirigeants de l'American College of Chest Physicians (ACCP), de l'ATS et de l'European Respiratory Society (ERS) pour échanger de l'information sur divers enjeux en santé respiratoire et pour planifier des collaborations à des initiatives précises. Les projets conjoints en cours incluent une collaboration nord-américaine à la Journée mondiale de la spirométrie, l'élaboration de lignes directrices conjointes sur les exacerbations aiguës de la maladie pulmonaire obstructive chronique avec l'ACCP, la présentation de notre programme scientifique annuel au congrès CHEST de l'ACCP, des exposés scientifiques lors d'événements comme le CCSR et le congrès mondial de l'asthme, la tenue du concours d'affiches de recherche de la SCT dans le cadre du congrès international annuel de l'ATS et le programme d'échange de la bourse de recherche conjointe Peter Macklem de la SCT/ERS. De plus, nous célébrerons bientôt l'entrée en poste de Darcy Marciniuk (ancien président de la SCT) à la présidence de l'ACCP, ce qui témoigne avec éloquence de notre collaboration sur la scène nord-américaine!

Nous participons également à des initiatives mondiales comme le Guidelines International Network et l'Alliance mondiale contre les affections respiratoires chroniques. Nous y sommes bien représentés par d'anciens dirigeants de la SCT, notamment Louis-Philippe Boulet, président du Comité des lignes directrices canadiennes en santé respiratoire.

Nos partenariats s'étendent jusqu'à notre revue scientifique. Sous la direction de Peter Paré, rédacteur en chef de la Revue canadienne de pneumologie (RCP), nous créons des protocoles d'entente avec la Société canadienne de soins intensifs et la Société canadienne du sommeil. Ainsi, nous visons aborder une gamme élargie d'enjeux cliniques et de recherche afin de rehausser le contenu et le lectorat de la RCP.

Le dernier élément, mais non le moindre, ce sont les partenariats que nous favorisons par le biais de la stratégie nationale de recherche 
partners in the development of the NRRS. Together with the Canadian Lung Association, who committed $\$ 1.085$ million over five years as matched funding for our joint application to create a national respiratory research network, they are helping to build respiratory research capacity in Canada and to ensure that the respiratory research community in Canada continues to 'punch above its weight'.

As the current president of the CTS, I fully support these partnerships built by my predecessors - I intend to make these relationships even stronger. The CTS's effectiveness in respiratory health can only be enriched by its collaboration with others. We won't go it alone that would not be best for respiratory health or the CTS.

Respectfully submitted, Robin G McFadden MD FRCPC President, Canadian Thoracic Society en santé respiratoire. Des facultés de médecine et des directeurs de division des quatre coins du pays, l'Institut de la santé circulatoire et respiratoire des Instituts de recherche en santé du Canada et AllerGen font partie des partenaires clés de l'élaboration de cette stratégie. En collaboration avec l'Association pulmonaire du Canada, qui s'est engagée à verser un financement de contrepartie de 1,085 million de dollars réparti sur cinq ans en réponse à notre demande conjointe en vue de créer un réseau national de recherche en santé respiratoire, ils contribuent à stimuler la capacité de recherche en santé respiratoire au Canada et à faire en sorte que la communauté canadienne de la recherche en santé respiratoire continue de jouer dans la cour des grands.

En tant que président de la SCT, j'appuie entièrement ces partenariats conclus par mes prédécesseurs, et j'ai même l'intention de les renforcer. L'efficacité accrue de la SCT en santé respiratoire passe par ses partenariats avec d'autres intervenants. Nous ne faisons pas cavalier seul. Ce ne serait ni dans l'intérêt de la santé respiratoire, ni dans celui de la SCT.

Salutations distinguées, Robin G McFadden, MD FRCPC Président, Société canadienne de thoracologie 


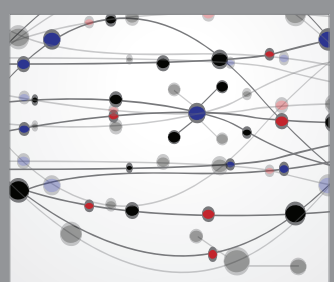

The Scientific World Journal
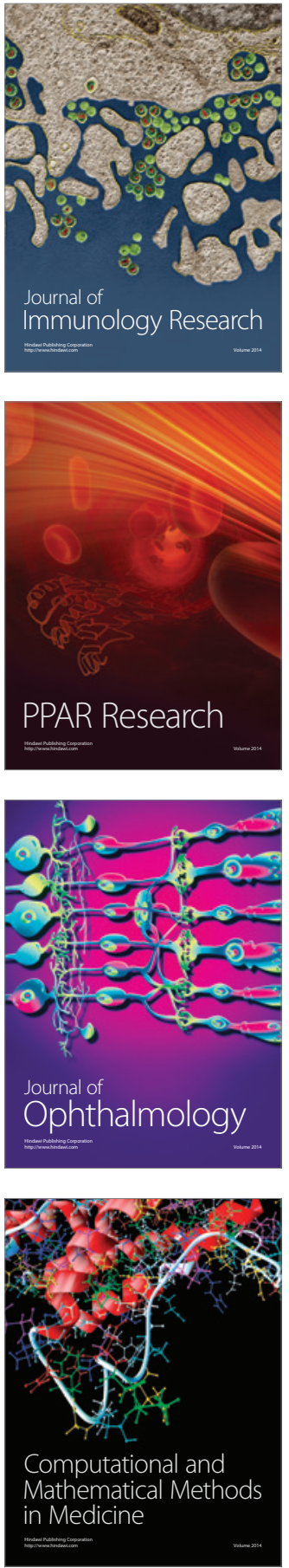

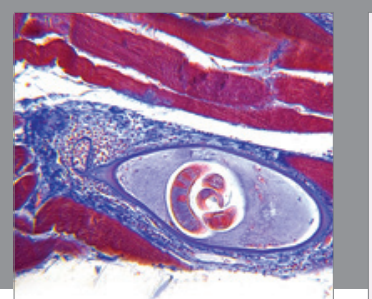

Gastroenterology Research and Practice

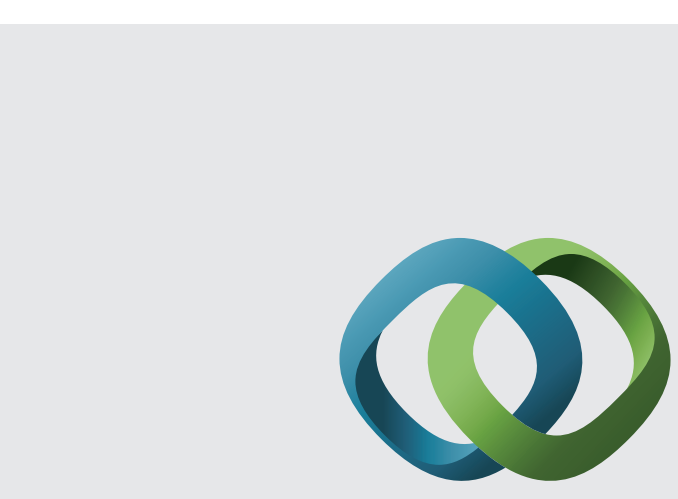

\section{Hindawi}

Submit your manuscripts at

http://www.hindawi.com
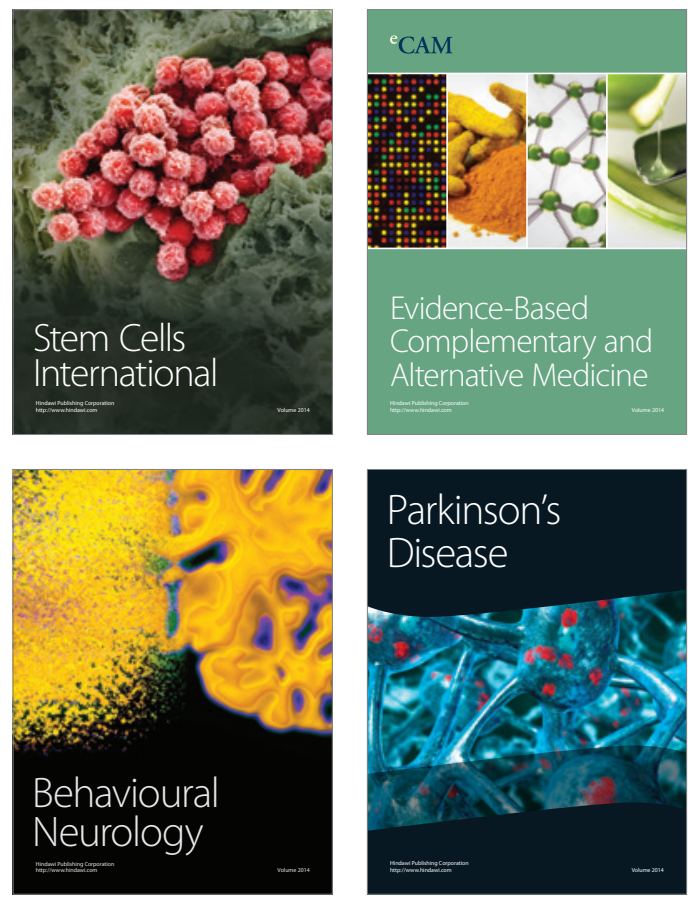
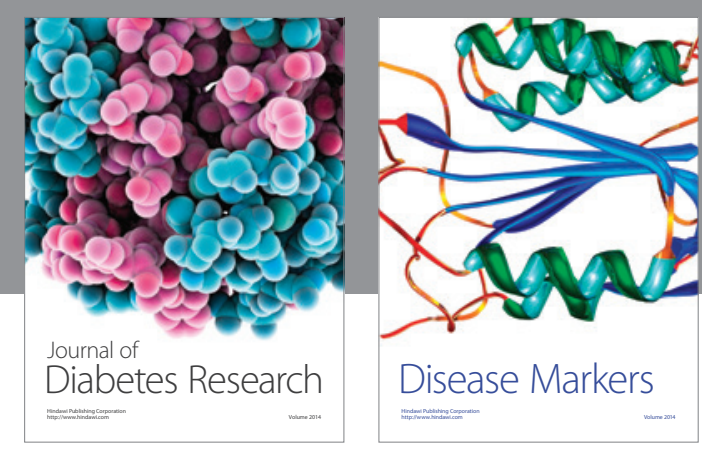

Disease Markers
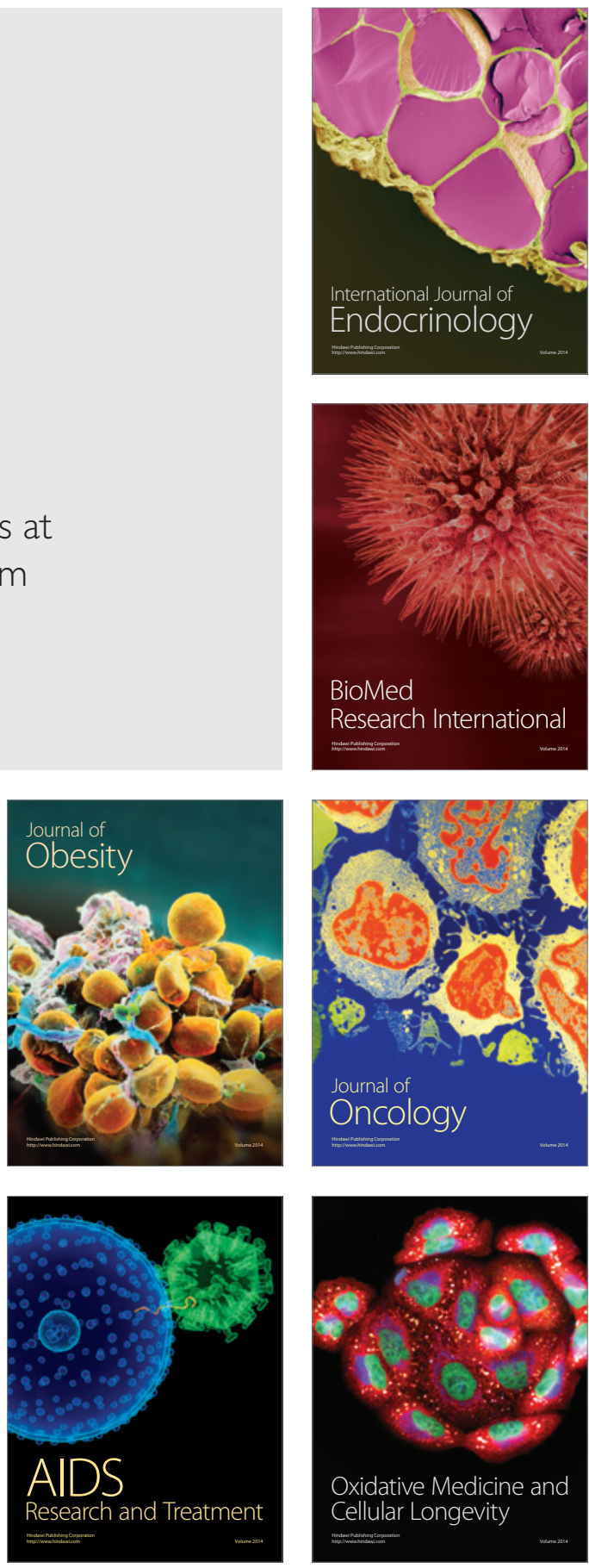